\section{SEPARATION OF ISOTOPES AND THERMAL DIFFUSION*}

\author{
By PROF. JAMES KENDALL, F.R.S. \\ University of Edinburgh
}

THE fact that the same element may exist in two or more forms, which are chemically identical even though their atomic weights differ, was first noted by Soddy (1910) in connexion with radioactive elements. A little later Sir J. J. Thomson (1913) showed, by the method of positive ray analysis, that neon, with an average atomic weight of $20 \cdot 2$, is a mixture of two kinds of atoms with masses 20 and 22 respectively. The brilliant extension of this method of attack by Aston established that most of the common elements are similarly inhomogeneous. Chlorine $(35 \cdot 457)$ is a mixture of ${ }^{35} \mathrm{Cl}$ and ${ }^{37} \mathrm{Cl}$; tin $(118 \cdot 70)$ exists in as many as ten forms, with atomic masses ranging from 112 to 124. These chemically identical species of the same element with diverse masses are known as isotopes.

The separation of isotopes has been strenuously attempted by many chemists during the last twenty-five years. There is only one instance where Nature has, in a sense, performed this separation for us lead derived from uranium disintegrations consists mainly of the isotope with mass 206, while lead derived from thorium disintegrations contains chiefly the isotope with mass 208 . In all other cases the proportions of the various isotopes of any particular element appear to be practically constant, whatever the source. Partial separation has proved possible, although exceedingly difficult, in several investigations; complete separation was first achieved in 1933 with hydrogen.

This lecture summarizes, in the main, a series of researches in this field which I and my co-workers have carried out since 1919.

\section{lonic Mobility}

A long-standing controversy has been waged on ionic mobility; one school insisting that ionic mobility is fundamentally dependent upon ionic volume, another being equally confident that it is fundamentally dependent upon ionic mass. The results available in the literature for homologous series of organic anions and cations have been utilized by both parties to prove their respective points; but, since we have no definite knowledge as to what amount of solvent accompanies any ion in its journey towards an electrode, and since it is the total mass or total volume of the ion and of its accompanying solvent envelope which must be taken into account, such data obviously offer us no means for definitely determining the problem.

The discovery that isotopes possess equal atomic volumes first put us in a position to impose a crucial test, for isotopic ions necessarily differ in mass. If mass is influential, therefore, it should be possible to obtain a separation of isotopes by taking advantage of the fact that the lighter ion will migrate more rapidly than the heavier. This idea of an 'isotopic race', however, cannot be carried out experimentally as simply as it might seem at first sight. Ions do not compete under the influence of the electric

- From a lecture at the Royal Institution, delivered on May 8. current in the manner of a track meet, unless we extend our experiences to imagine a continuous relay race. We cannot start all of our ions at one point and obtain a separation by noting when those of a certain species have passed a given goal, for there must be maintained a steady supply all the way from one electrode to another in order for the current to pass, and it will not help us much if a faster ion hurries ahead of its slower neighbour, since it will merely find itself in the company of other slower ions which happened to start a little in advance. By a modification of the experimental procedure, nevertheless, our 'isotopic race' may be converted into a 'parade' which can be suitably regulated.

The apparatus used is shown in the accompanying diagram, and its applicability may be illustrated by a condensed description of the technique employed in the case of chlorine.

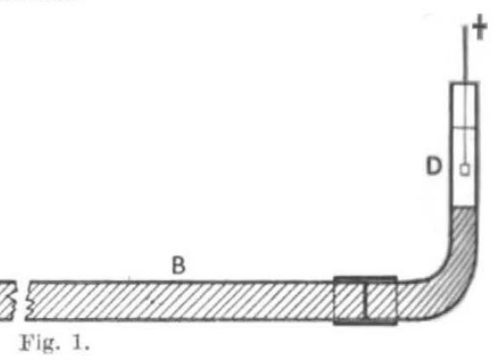

An agar-agar gel $A$ containing sodium chloride is inserted as a short middle section in a long horizontal tube of Pyrex glass, one and a half inches in internal diameter. On one side of the chloride gel is added a gel $B$ containing sodium hydroxide; on the other side a gel $C$ containing sodium acetate. The ends of the tube are connected with right-angled Pyrex bends of the same diameter, and the gels continue well up into these bends, as in the diagram. Above the hydroxide gel, after it has set, is poured concentrated sodium hydroxide solution $D$, and above the acetate gel a solution $E$ of sodium acetate in concentrated acetic acid. Platinum electrodes are placed in these two solutions, and a current is passed through the tube, the electrode in $D$ being made the anode and the electrode in $E$ the cathode. The heating effect of the current upon the gel may be minimized by submerging the tube in a long trough filled with running tap-water.

At the beginning of the run, the boundary surfaces between the various sections of the gel are perfectly sharp. When the current is turned on, the boundaries move towards the anode. Inasmuch as there is a faster-moving ion in front of the chloride ion, and a slower-moving ion behind it, admixture of the salts is absolutely prohibited and the boundaries remain distinct throughout the whole experiment. In order to maintain the width of the chloride section approximately constant, it is well to arrange the concentrations of the various salts in their respective gels in accordance with the transference numbers of their anions. Even if this is not done exactly, however, the boundary concentrations soon automatically adjust themselves to the required ratios.

The rate at which the boundaries move depends upon the potential drop between the electrodes, the length of the tube, and the concentrations of the solutions. In actual practice, the horizontal tube is made up of several three-foot sections connected by rubber bands, and the current is regulated (100-500 volts) so that the boundaries advance about 12-18 in. 
a day. When the front chloride boundary has almost reached the end of the tube, the apparatus is taken apart. The two rear sections are discarded, two new sections filled with hydroxide gel are inserted in front of the chloride, and new bends are fitted on as before. The chloride ions are now forced to migrate into these two new sections, and the whole procedure is repeated until they have progressed through about $100 \mathrm{ft}$. of the gel. The chloride gel is then removed from the tube and immediately cut up into strips about $1 \mathrm{~cm}$. in width.

If, now, the isotopic chloride ions with atomic masses 35 and 37 respectively possess significantly different mobilities, the front segments of the gel will contain only the faster-moving and the rear segments only the slower-moving isotope. Preliminary experiments in which a mixture of two sodium salts with anions of known mobilities was substituted for the middle sodium chloride section fully demonstrated this point. Thus, when a mixture of sodium iodide and sodium thiocyanate was employed and the boundary was moved only a few feet, the front segments were found to contain only sodium iodide and the rear segments only sodium thiocyanate. The observed lag of the slower ion was almost exactly equal to that calculated from the difference in mobilities. In this particular case the difference is approximately 16 per cent. In subsequent experiments this difference was narrowed by choosing other suitable pairs of ions: for example, barium and calcium, barium and strontium, and iodide and chloride. The differences in mobilities are here reduced to 8 per cent, 5 per cent and 1 per cent respectively, and the results obtained showed that the method could be depended upon to provide a very considerable degree of separation even at the lowest limit tested.

It was highly disappointing, therefore, to be forced to admit from the results of all our experiments with isotopic ions that no significant separation could ever be detected. The only conclusion that can logically be drawn is that the mobilities of isotopic ions are well within 1 per cent of equality, and that those workers are substantially justified who contend that volume is the decisive factor in determining ionic mobilities. Later theoretical advances, it must be added, support this view, although the matter is still not absolutely settled (Jette, 1927).

Although all the work on isotopes, as has been stated, led to no successful separations, yet the positive results obtained in the test experiments on known mixtures did inspire the hope that the ionic migration method might, after all, prove of practical service in the separation of more familiar materials which are ordinarily obtained by the chemist in a pure state only with extreme difficulty. The most important example of this type is furnished by the family of the rare earth metals. The group of elements known as the rare earths comprises the elements of atomic number 57 to 71 inclusive, and yttrium with atomic number 39 . These elements are distinguished by such extraordinary alikeness in chemical and most physical properties, due to the identical arrangements of the two outer shells of electrons in their atoms, that they actually approach isotopes in similarity. It is necessary, in practice, to take advantage of slight differences in solubility observed for corresponding salts and to resolve a given mixture into its components by a long series of fractional crystallizations or precipitations, ranging in number from several hundred to many thousand, according to the elements present and the degree of purity desired. These operations are so laborious and time-consuming that chemically pure samples of individual rare earth compounds are practically unknown, save on the shelves of a few skilled workers in the field of atomic weights. The 'rareness' of the rare earths is due not so much to the lack of abundance of their ores in Nature as to the lack of a simple method for their separation.

The first separation attempted was upon a mixture of yttrium and erbium, kindly furnished by Prof. James, of the University of New Hampshire. Potas. sium was used as a preceding faster ion and trivalent chromium as a following slower ion. Not only could the position of the rear boundary be more conveniently followed in this particular case because of the colour of the chromic solution, but it was also found possible to observe the actual progress of the separation within the rare earth section in a very simple way by means of a small direct vision hand spectroscope. The majority of the rare earths give solutions with characteristic absorption spectra, and, by noting the increase or decrease in intensity of the most prominent lines in various parts of a section, the experimenter could immediately detect any change in composition in the whole length of the section without interrupting the run. Here yttrium rapidly accumulated in the front half of the section and erbium was relegated to the rear. After a run of fourteen days, during which the boundaries moved two metres, an almost perfect separation had been effected.

The next mixture tested consisted of neodymium and praseodymium, two elements which are so nearly alike that their first differentiation by Otto von Welsbach still forms one of the triumphs of technique in this difficult field. Neodymium salts exhibit a purple solution in water, while praseodymium salts give a green solution. A beautiful crystal of neody. mium nitrate and an equally fine specimen of praseodymium sulphate were secured from the Chandler Chemical Museum of Columbia University and the careful work of several years was deranged in a few minutes by mixing these two salts to obtain a solution with an intermediate neutral tint. With this solution as a central section and with the same arrangement as in the preceding experiment, it required only a few days migration to disclose the fact that the front portion of the section was becoming distinctly green and the rear portion purplish. Observation by means of the spectroscope simultaneously showed that the characteristic absorption lines of praseodymium were becoming more pronounced in the front section and fading out in the rear, with the opposite behaviour for the neodymium. At the end of ten days, substantial progress towards a final separation had been accomplished.

Experiments with other mixtures of rare earths showed that, while complete separation could not be secured in every case, yet in most mixtures the mobilities of the ions were sufficiently divergent to compel a very rapid concentration of one particular component in the front or in the rear section. The method may, therefore, be considered as a general new method for obtaining pure samples of the rare earth elements with the expenditure of much less time and trouble than is required by the classical method of fractional crystallization (see, however, Selwood and Hopkins, 1929).

Important industrial uses for the rare earth elements will undoubtedly be discovered as soon as 
more convenient means for their isolation are developed. Aluminium remained a chemical curiosity until this same end was achieved, and while the later members of the aluminium family may not duplicate the successful career of their brilliant brother, yet it would indeed be strange if Nature had omitted to endow them, alone of all the elements, with no properties of service to mankind.

The success of the experiments with rare earths suggested that the method might be applicable to the separation of radium from barium. The concentration of radium from the barium residues of carnotite ores at present involves a very tedious series of fractional crystallizations, and, since it had already been found that barium could be separated from the other elements of the alkaline earth family by the method here under discussion, it appeared very probable that a similar separation from radium, the last member of this same family, could also be accomplished. Samples of barium residues containing known amounts of radium and of mesothorium (an isotope of radium) were obtained and, after a few days migration, the sections were cut into segments and their radioactivity examined. It was found that the radioactive components of each mixture tested accumulated very rapidly in the front part of the section. The ease of the separation in this case is so striking as to suggest that the ionic migration method may come into technical use for the concentration of radium in barium residues

An attempt to separate hafnium and zirconium did not lead to such conclusive results. Hafnium exists to the extent of several per cent in all zirconium ores, and the similarity in properties is so pronounced that the actual discovery of hafnium was not definitely established until 1923. A sample of hafnium-rich zirconium oxide was kindly furnished us by Prof. Hevesy. The elements were not amenable to separation by the ionic migration method in the form of positive ions, owing to hydrolysis, and only after considerable search was a suitable negative complex ion discovered in the form of a complex oxalate. After long migration, analysis showed a very slight accumulation of hafnium in the rear of the section, the mobilities of the complex anions being evidently so close together as to render a complete separation impracticable.

The results in an entirely different field have been of better promise. Many of the alkaloids particularly useful for medicinal purposes are derived from natural sources as mixtures of several individual members which can be separated by the ordinary methods of organic chemistry only with great difficulty. The alkaloids, however, are weak bases which form soluble hydrochlorides, and the mobilities of the cations of these salts are not identical. It should, consequently, be possible to obtain a pure sample of an especially valuable alkaloid from the mixture of similar materials with which it naturally occurs by use of the ionic migration method, and several preliminary experiments indicate that the method is indeed applicable in a majority of cases.

\section{Ionic Discharge Potential}

Isotopic ions differ only in their minute nuclei, and these are surrounded by a relatively distant cloud of external electrons, increasing in number as we proceed up the periodic system. When an ion is discharged at one of the electrodes during the process of electrolysis, all that happens is that one or more electrons is added to, or taken from, this external cloud. Although, therefore, the free energy changes involved in the discharge of two isotopic ions are not necessarily identical, it is unlikely that the discharge potentials will be markedly different in the two cases, when expressed in volts. If we could pass an electric current through a solution containing two isotopic ions, adjusting the voltage so that the decomposition potential of one species was just exceeded while the decomposition potential of the other species was just not attained, then a perfect separation should be feasible. Owing, however, to the impossibility of adjusting the voltage with sufficient delicacy, and to the fact that reverse interaction of the discharged material with the solution, unless its immediate withdrawal from the system was effected, would probably tend to re-establish equilibrium conditions fairly rapidly, it appeared that the utmost that would be attainable in practice would be an 'electrolytic fractionation'.

Encouragement that a partial separation might be achieved by this method was derived from the knowledge that it is possible to vary the composition of a tin-lead alloy, obtained by electrolysis of a mixed solution of tin and lead salts, very significantly by varying the voltage employed. The discharge potentials of tin and lead ions differ by approximately 0.01 volts, so that it seemed that there was a likelihood of being able to establish some slight degree of separation of isotopic ions by fractional electrolysis, even if their discharge potentials were still more nearly identical.

The choice of an element to test the method presented great difficulty. Other things being equal, a light element would obviously be preferable, since the external electrons are fewer in number and shield the nucleus less effectively. The experimental obstacles in the case of lithium, the lightest known isotopic element at the time when this work was started (1921), are, however, exceptionally grave, owing to the activity of the free metal and to the elaborate technique necessary for its exact atomic weight determination. Other light elements presented similar objections, and the selection finally fell upon mercury. It is true that mercury is very near the wrong end of the periodic system, its isotopes ranging in atomic mass from 197 to 204 , but the free metal is inert and the smallest change in its average atomic weight can be immediately and readily detected by means of density determinations. It was consequently decided to attempt the fractional electrolysis of mercury first, and to continue with lithium later, if the results obtained were sufficiently promising.

The results obtained have been presented in detail in a Ph.D. thesis from Columbia University (Haring, 1924). Electrolysis of an acid solution of mercurous nitrate, using a low voltage and low current density, gave mercury with a density only 0.999981 that of ordinary mercury. This value was the average of fourteen different electrolyses. Parallel experiments in which a high voltage and high current density were employed gave mercury with density unchanged.

The reduction in density, it will be seen, was only nineteen parts per million, which represented three times the average deviation of the individual results. The greatest difficulty encountered in the whole investigation was the exact setting of the mercury level in the pycnometers and, although the error involved thereby was minimized by repeating each 
setting five times and averaging the results of the five weighings, the probable error involved still amounted to $7 \cdot 5$ parts per million. This fact, together with the disappointingly small change $(0.004$ unit) in the average atomic weight of the electrolysed mercury, discouraged us from proceeding further at the time.

I have always been convinced, however, of the reality of the fractionation indicated and, in the expectation that more conclusive results would be obtained with a lighter element than mercury, $\mathbf{M r}$. McLaren of Syracuse University began a systematic study of the electrolytic method of isotopic fractionation under the direction of myself and Dr. Ludlam at the University of Edinburgh in 1930. A considerable amount of work had been done on lithium, but no final results attained, when the discovery of the isotopic character of hydrogen turned our attention early in 1932 to that element, which obviously offered still greater chances of success. Spectrographic evidence suggesting that the proportion of the heavier hydrogen isotope in the first fraction of the electrolysis of water was less than in ordinary hydrogen had been obtained when parallel investigations in the United States were brought to a successful conclusion. The isolation of pure 'heavy' hydrogen and the preparation of pure 'heavy' water from the final fractions of the repeated electrolysis of ordinary water must be regarded as one of the most significant achievements in chemistry in recent years. It is disappointing to have been anticipated, but there is some consolation available in being the first to employ the method by which the separation was made.

\section{Thermal Diffusion}

Since 1933, striking advances in the separation of the isotopes of heavier elements have been made, notably by the chemical exchange method of Urey, but perhaps the most promising general line of attack is that opened up recently by the work of Clusius and Dickel on thermal diffusion.

This property was originally noted by Ludwig in liquids so long ago as 1856. Ludwig placed a solution of sodium sulphate in a U-tube, one limb of which was kept in boiling water and the other in meiting ice, and found that after two days solid crystals had separated out in the cold limb, while the concentration in the hot limb had decreased to one half. Soret (1879) made measurements of the same kind with various salts, employing vertical tubes with the upper part heated and the lower part cooled, and his results were utilized, not very justifiably, by van 't Hoff (1887) in confirmation of the applicability of the gas laws to solutions.

Thermal diffusion in gases was first discussed by Enskog (1911) and predicted independently by Chapman (1917). In the latter year, Chapman and Dootson achieved a partial separation of a mixture of carbon dioxide and hydrogen contained in two glass bulbs connected by a capillary tube, with a temperature difference of $210^{\circ}$. The theory of the process, both for gases and for liquids, however, remained obscure, as will be evident from the fact that Chapman, in 1929 , pointed out that existing theories did not even indicate with any certainty the sign of the slight amount of fractionation generally obtainable.

Only in 1938 did thermal diffusion suddenly come into practical prominence through the astoundingly simple discovery that the substitution of a horizontal for a vertical temperature gradient enhanced the effect to an enormous degree. In the older forms of apparatus, convection currents were deliberately avoided, but Clusius and Dickel ingeniously used such currents to assist the normal thermal diffusion process by what has been called 'thermo-syphon action'.

This action is illustrated in the accompanying sketch, where the convection currents set up in a narrow rectangular vessel with opposite heated and cooled surfaces are indicated by arrows. Normal thermal diffusion establishes a small concentration gradient in the direction $A B$, the concentration of the lighter component of the gas or solution contained in the tube increasing at $A$ and decreasing at $B$. The upward convection current consequently causes the ascent of excess lighter mole. cules, the downward current the descent of excess heavier molecules. A second concentration gradient is thus developed in the direction $C D$, and the steady continuation of the process may ultimately establish, under suitable conditions, a complete separation of the two components of the original mixture.

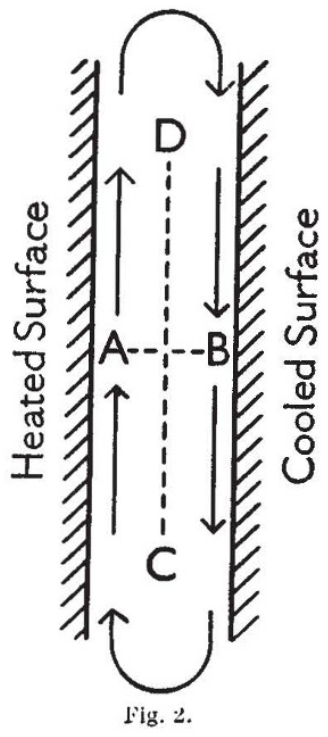

An improved form of apparatus devised by Clusius and Dickel for use with gases consists of a long vertical glass tube, externally cooled, with an electrically heated platinum or nichrome wire running down its centre. With a series of successive tubes, altogether 36 metres in length, Clusius and Dickel succeeded in obtaining from ordinary hydrogen chloride top samples corresponding with a $99 \cdot 6$ per cent content of the lighter isotope of chlorine and bottom samples corresponding with a 99.4 per cent content of the heavier isotope. This represents so far the only reasonably complete separation secured by the thermal diffusion method, but the applicability of the method to other cases is obvious.

Considerable work, though practically all of a preliminary nature, has also been done on thermal diffusion in liquids since 1938, and I shall conclude with an account of some simple experiments carried out in my own laboratory by Taylor and Ritchie. The solutions employed are enclosed in a vertical annular space, less than $0.5 \mathrm{~mm}$. in diameter, between two concentric glass tubes, approximately 1.5 metres long. A temperature gradient of about $80^{\circ}$ is maintained across this space by the passage of a current of cold water through the inner tube and a current of steam through a surrounding jacket. Eight hours of thermal diffusion of a solution of copper sulphate fractionates it so thoroughly that a goodly crop of crystals will separate out at the lower end, while the characteristic blue colour of the salt will entirely disappear from the uppermost section. Two hours of thermal diffusion of a solution of copper bromide suffices to develop the characteristic reddish-brown concentrated coloration and the bluish dilute colour at opposite ends of the tube. A solution of cobaltic chloride after two hours of thermal diffusion clearly exhibits the deep blue and pink of concentrated and dilute solutions at its upper and lower ends. 
It will be evident that thermal diffusion represents a most valuable new tool at the disposal of the chemist, facilitating separations of a most diverse character. One practical point that has already come up for investigation is the influence of thermal diffusion on ingot segregation in steels. Metals are such good conductors of heat that it seems unlikely that any steep temperature gradient could persist for a sufficient length of time in the liquid contained in an ingot mould for thermal diffusion to induce significant segregation. At the suggestion of $\mathrm{Dr}$. C. H. Desch, however, an experiment to test this point was undertaken in my department by Dr. Ritchie. The apparatus employed was similar to that already described, but the annular distance was reduced to $0.25 \mathrm{~mm}$. and the length of the concentric tubes increased to 2 metres. The annular space was filled with a solution of 1.5 per cent zinc (density $7 \cdot 1$ ) in mercury (density $13 \cdot 6$ ). Even under such favourable conditions, no fractionation whatever was observable after a run lasting for 32 hours.

This negative result is no doubt largely due to the high thermal conductivity of the amalgam, which reduces the temperature gradient by making the drop between the annular liquid surfaces much less than that between the surrounding glass walls. The same factor, however, as has been noted above, should also diminish the effect of thermal diffusion in ingots. Further work in the field is obviously necessary, but in the meantime the view expressed by Desch that thermal diffusion cannot play an appreciable part in producing the observed segregation in steel ingots appears to be fully justified.

\section{FUTURE OF THE POPULATION OF GREAT BRITAIN}

\section{BY COLONEL SIR CHARLES ARDEN-CLOSE, K.B.E., C.B., C.M.G., F.R.S.}

A N interesting memorandum, entitled "Current A Trend of Population in Great Britain", was presented to Parliament by the Minister of Health and the Secretary of State for Scotland in May 1942*, but it is explained, in the opening paragraph, that this memorandum was actually "submitted shortly before the outbreak of the war by the RegistrarsGeneral of England and Wales and of Scotland to the Royal Commission on the Geographical Distribution of the Industrial Population". It was not included in the papers published by that Commission. The facts dealt with are those available up to April 1938 and no account is taken of the War.

The memorandum is in the form of a compact little pamphlet of twelve pages, with tables and two diagrams. The account is somewhat compressed and requires attentive study. It brings our knowledge of the facts reasonably well up to the date mentioned, and, with the aid of certain assumptions, gives indications of what our numbers might be twenty or thirty years hence; but it has to be borne in mind that the assumptions do not include the possibility of war.

The main intention of the memorandum is stated to be the presentation of the facts in as objective a manner as possible, so as to correct misunderstandings based on rather sensational forecasts as to

* H.M. Stationery Office, 1942. 2d. net. what might be the fate of the population of Great Britain a century or so hence. A warning is given that forecasts extending to such a distant future have very slight value.

It is stated that, "as long ago as 1926, attention began to be drawn in official reports to the significance of the phase which the falling birth-rate was then entering". The crude birth-rate, which reached its maximum of $\mathbf{3 5 . 4}$ per thousand of the population in the period 1871-75, after that showed a continuous decline until the year 1933 when it reached the low figure of 14.7, "from which time the fall appears to have been arrested and a minute, though so far uninterrupted, reverse of the movement taken its place". The estimated figure given for 1938 was $15 \cdot 5$, but actually it was $15 \cdot 1$. We can bring the figures up to date, thus : $1939,14 \cdot 9 ; 1940,14 \cdot 6 ; 1941,14 \cdot 2$. These figures are later than the date of preparation of the pamphlet; they are obviously affected by the War and will not serve as a guide to future peace-time rates, for it must be remembered that great wars, such as the War of 1914-18, or the present War, do materially diminish the populations of the countries engaged in them ; the population of Great Britain was probably some two millions less at the time of the census of 1921 than it would have been had there been no war a few years before.

A table is given to show the average annual percentage of increase of our population, and from this it appears that between 1871 and 1911 the average annual increase was $1 \cdot 12$, but after 1911 there was a marked drop and the average increase up to 1938 was about $0 \cdot 45$. The most striking drop was from 0.98 in $1901-11$ to 0.46 in $1911-21$, that is, the most marked change occurred in the war period "when the rate of increase, which, prior to that time had been of the order of 1 per cent per annum, dropped to rather less than half that figure, at which level it has been maintained ever since".

It is well known that since 1923 the birth-rate has not been sufficient to provide a stationary population ; in fact the births are only, by Dr. Kuczynski's calculation, about three quarters of the number required to reach that standard, in spite of the present continued increase of our numbers. Later information puts the net reproduction-rate, in 1938, as 0.84 , so that fertility would have had to be raised by about one fifth to result in a stable population. To illustrate the "transitory and unbalanced nature of the present age distribution" a comparison is made between this and two theoretical stationary populations, resulting in each case from a constant annual number of births of 700,000 , with different rates of mortality. It is stated that the comparisons show that the population would be maintained at or near its present level of forty-six millions if there were continued the flow of 700,000 births which were registered in 1937. But, of course, the supposition of a continued, constant flow of this kind "could only be realized by a gradual rise in fertility over the next two or three decades". It is added that such a recovery might possibly be secured "with the aid of measures ensuring a national population policy".

The male section of the important group $15-45$ is now much smaller than the female section of that group. It is possible that this inequality is not sufficiently stressed by demographers. The marriagerate must be adversely affected by such a disparity. We all of us know of women who have lost their prospective husbands in the War of 1914-18, and of others who found themselves in a world which 